\title{
Supplementary Material for Multi-model dynamic climate emulator for solar geoengineering
}

\author{
Douglas G. MacMartin, Ben Kravitz
}

June 12, 2016

The list of climate models that participated in the Geoengineering Modeling Intercomparison Project (GeoMIP) and are used here is given in Table S1; Figure S1 illustrates the GeoMIP G1 and G2 simulations that are used to construct and validate the emulator respectively. Several additional emulator results are given in Figures S2 and S3, comparing the simulated and emulated temperature and precipitation differences between land and ocean. Figure S4 illustrates for one model the emulator capturing of the first few principal components of the spatial temperature response. Figure S5 illustrates the ability of the dynamic emulator to capture modeled changes in Net Primary Productivity (NPP); changes in global-mean NPP are relatively linear in these climate models, and relatively unaffected by a solar reduction.

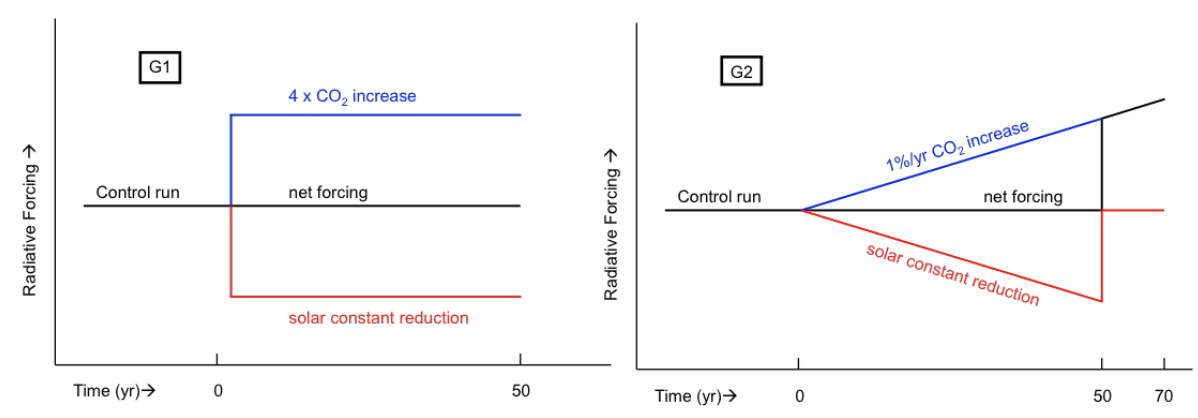

Figure S1: Schematic of GeoMIP G1 and G2 simulations. 


\begin{tabular}{l|c|}
$\begin{array}{l}\text { Climate } \\
\text { Model }\end{array}$ & $\begin{array}{c}\text { Solar Reduction } \\
\text { for G1 }(\%)\end{array}$ \\
\hline CanESM2 & 4.0 \\
CESM-CAM5.1-FV & 4.7 \\
GISS-E2-R & 4.5 \\
HadCM3 & 4.1 \\
HadGEM2-ES & 3.9 \\
IPSL-CM5A-LR & 3.5 \\
MIROC-ESM & 5.0 \\
MPI-ESM-LR & 4.7 \\
CSIRO-Mk3L-1.2 & 3.2
\end{tabular}

Table S1: Climate models used here, with the solar reduction $g_{4 \times}$ used in each model to compensate for $4 \times \mathrm{CO}_{2}$
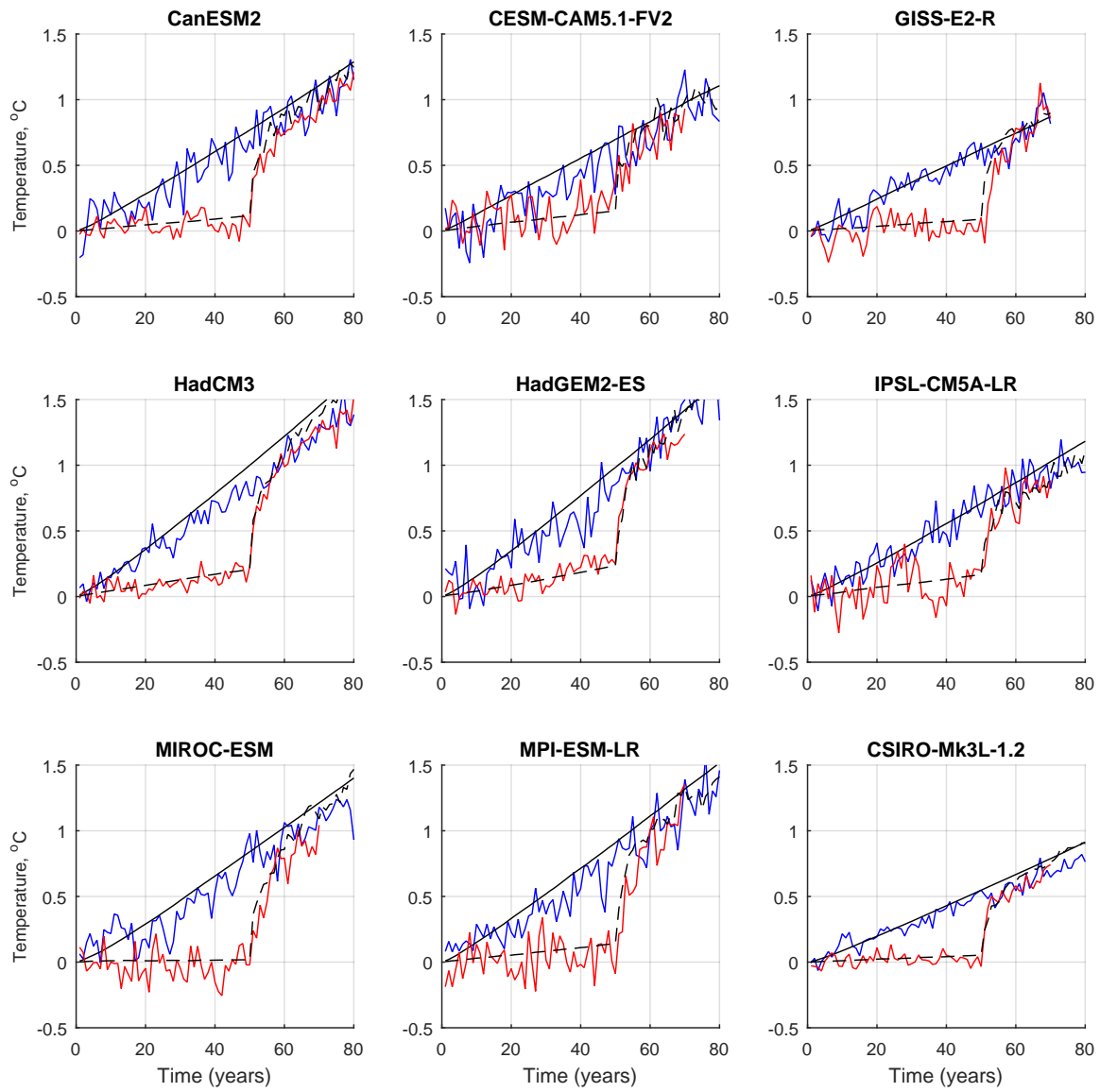

Figure S2: As in Figure 2 but for difference between average temperature over land and average temperature over oceans, for $1 \%$ per year increase in $\mathrm{CO}_{2}$ and GeoMIP experiment $\mathrm{G} 2$ for each of the climate models considered here. 

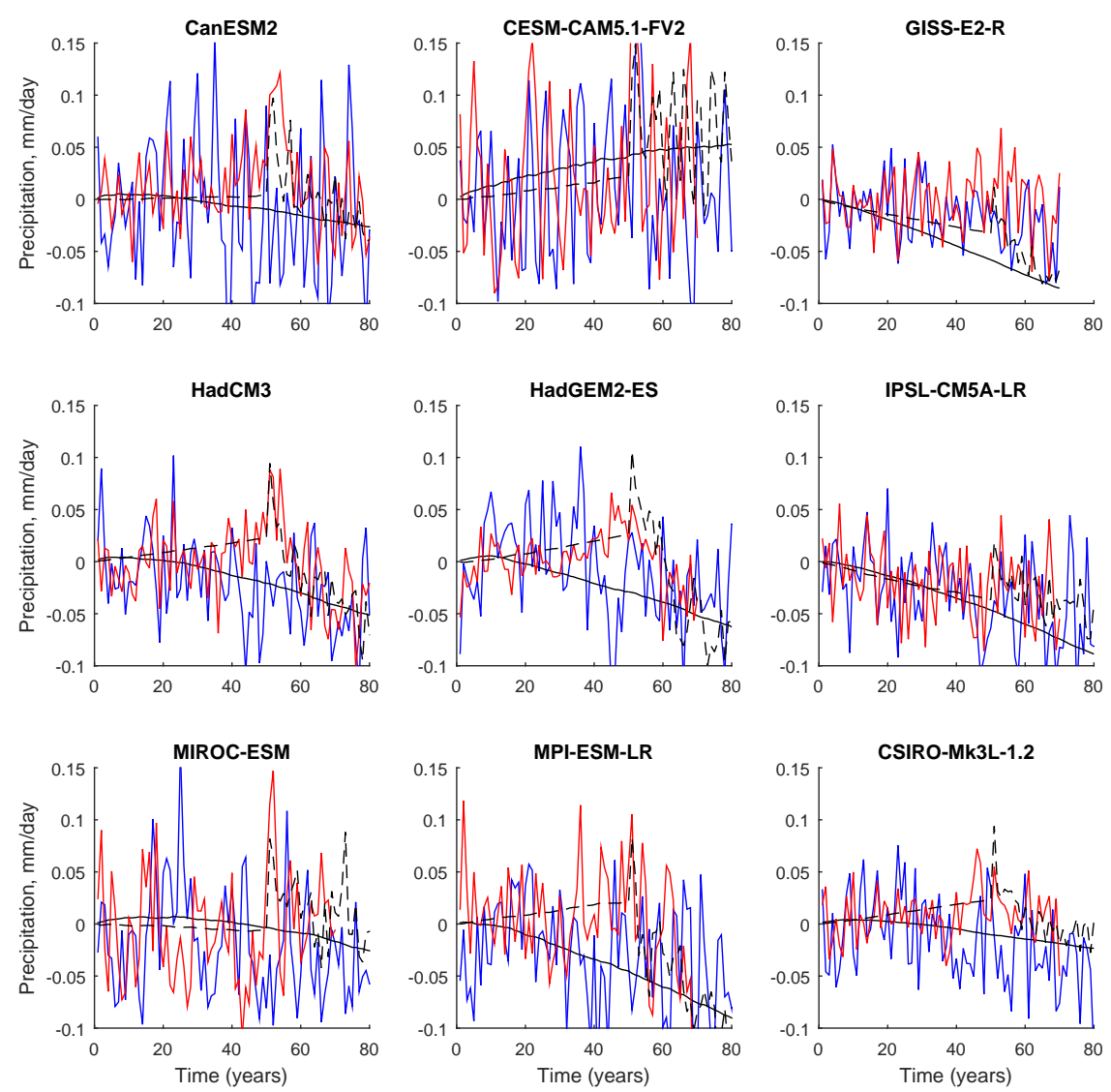

Figure S3: As in Figure 3 but for difference between average precipitation over land and average precipitation over oceans, for $1 \%$ per year increase in $\mathrm{CO}_{2}$ and GeoMIP experiment G2 for each of the climate models considered here. 

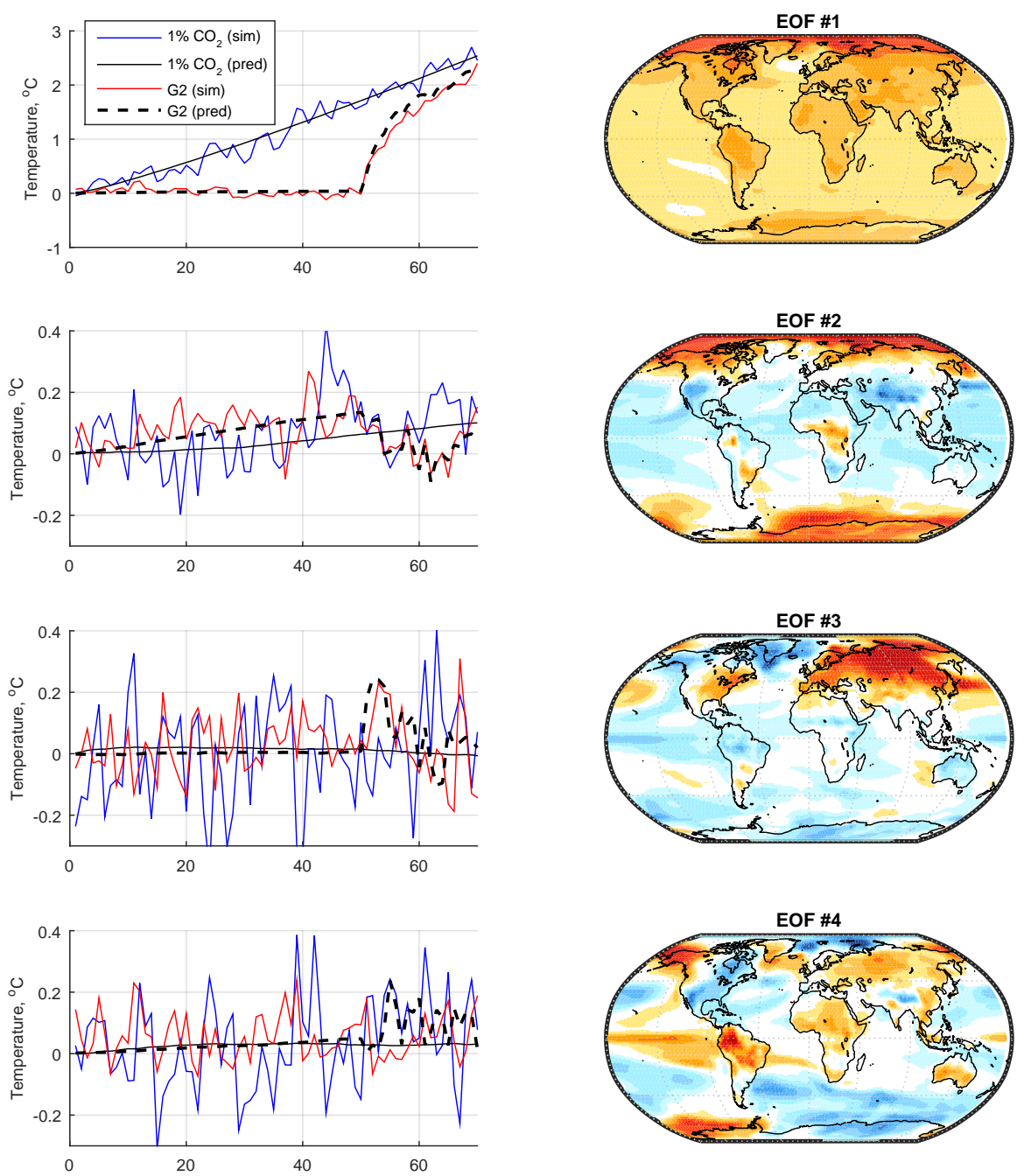

Figure S4: First few temperature EOFs for CanESM (right column), and the simulated and emulated time-history of the projection onto these EOFs for both $1 \%$ per year $\mathrm{CO}_{2}$ increase and G2 simulation for CanESM; other models give broadly similar results. The first EOF here gives the pattern of warming from $\mathrm{CO}_{2}$, while the second captures most of the difference in the response between $\mathrm{CO}_{2}$ and solar forcing. In this model, higher EOFs are primarily describing natural variability rather than forced response. 

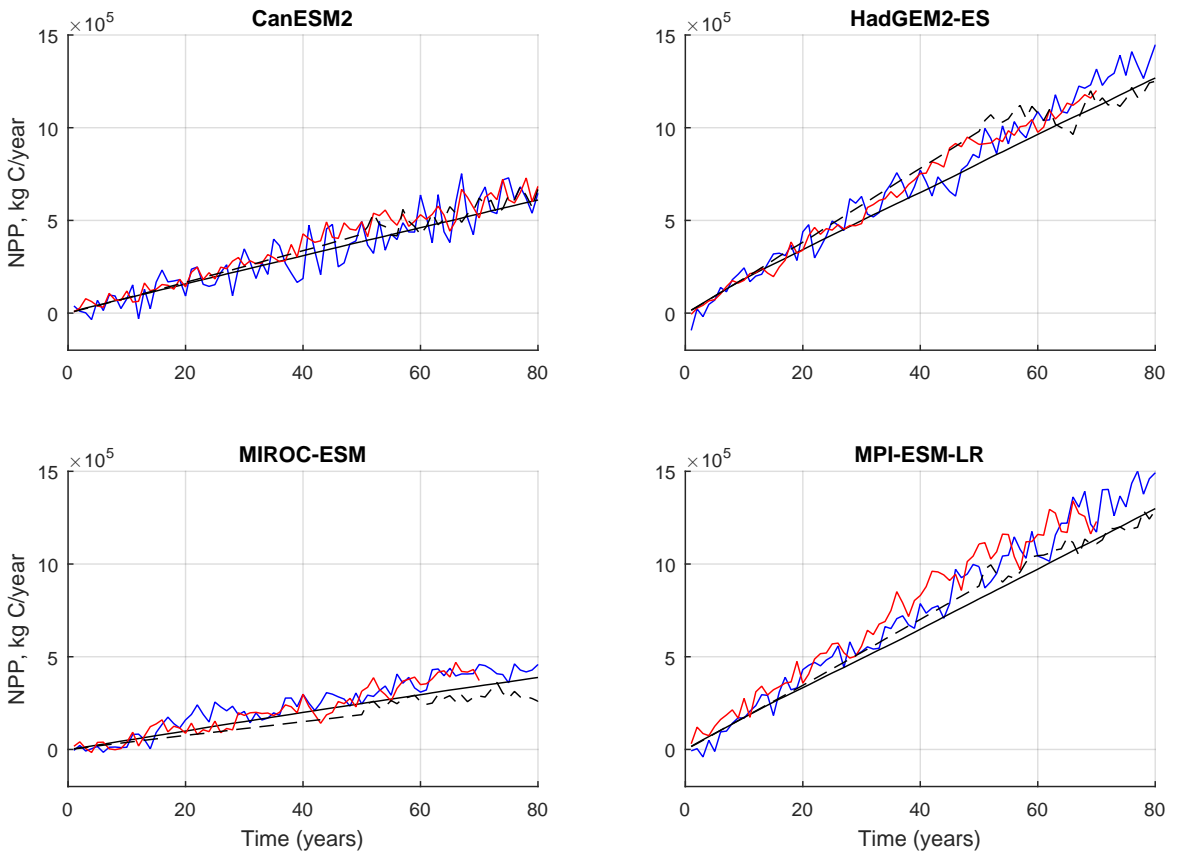

Figure S5: As in Figure 2 but for global mean net primary productivity (NPP). Simulated and emulated response are shown for $1 \%$ per year increase in $\mathrm{CO}_{2}$ and GeoMIP experiment G2 for several of the climate models considered here (NPP was not available for all models). 